\title{
Sympathikusdenervation und Baroreflexaktivierung Interventionelle Verfahren zur Hochdrucktherapie noch in der Prüfphase
}

\begin{abstract}
Mit dem Einzug der interventionellen Therapieverfahren scheint sich in der Hypertensiologie eine Entwicklung abzuzeichnen, wie sie die Kardiologie schon seit über einer Dekade höchst erfolgreich erlebt.
\end{abstract}

CardioVasc: Welches Potenzial steckt Ihrer Meinung nach in der renalen Sympathikusdenervation und der Baroreflexaktivierung?

Kreutz: In der Tat befinden sich mit der renalen Sympathikusdenervation (RSD) und der Baroreflexaktivierung (BRA) zwei interessante interventionelle Verfahren zur Hypertoniebehandlung in der klinischen Entwicklung. Vollkommen neu sind interventionelle Ansätze zur Hypertoniebehandlung ja nicht. Die bislang publizierten Studien belegen die prinzipielle Machbarkeit und das Potenzial sowohl für die RSD als auch die chronische BRA für die Blutdrucksenkung bei Therapieresistenz. Für die RSD wurde erstmalig in 2009 eine Proof-of-Concept-Studie (Simplicity HTN-1) entsprechend einer Phase-I-Studie vorgestellt, und Ende letzten Jahres wurde die Nachfolgestudie Simplicity HTN-2 bei der AHA-Tagung in Chicago präsentiert und zeitgleich in Lancet publiziert. Symplicity HTN-2 ist die erste randomiserte Wirksamkeitsstudie (Phase-II-Studie) zur RSD bei therapieresistenter Hypertonie. Das Verfahren war in spezialisierten Zentren unter Studienbedingungen sicher anwendbar.

ICardioVasc: Wie beurteilen Sie die Ergebnisse zur RSD?

Kreutz: Unklar und kritisch zu bewerten bleibt der Befund, dass die systolische und diastolische Blutdrucksenkung nach RSD in der ambulanten 24-Stunden-Blutdruckmessung ( $A B D M$ ) nach sechs Monaten mit -11/-7 $\mathrm{mmHg}$ deutlich schwächer ausfiel als bei den Praxismessungen (-32/-12 mmHg). Viele prinzipielle Fragen zur RSD können mit dieser ersten randomisierten Wirksamkeitsstudie naturgemäß noch nicht beantwortet werden.

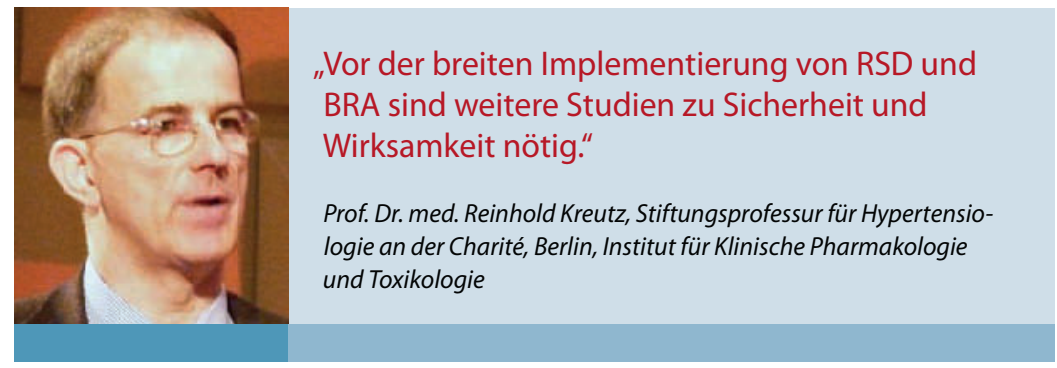

Diese betreffen u. a. die Langzeitwirksamkeit und-sicherheit, die Relevanz einer Reinnervation, Kosteneffektivität durch tatsächliche Einsparung von Medikamenten, die rationale Selektion und Überwachung geeigneter Hypertoniepatienten nach RSD im klinischen Alltag und die Übertragbarkeit auf andere Erkrankungen wie zum Beispiel auf Patienten mit chronischer Niereninsuffizienz.

ICardioVasc: Und wie sieht es bei der elektrischen Stimulation des Baroreflexes aus? Kreutz: Die BRA befindet sich noch in einem frühen Stadium der klinischen Entwicklung. Bei Anwendung dieses Verfahrens wird über ein implantiertes System der Baroreflex durch dauerhafte elektrische Stimulation des Karotissinus aktiviert und damit der Blutdruck gesenkt. Im vergangenen Jahr wurden die Ergebnisse der DEBUT-HT-Studie (Device Based Therapy in Hypertension Trial), einer europä- ischen "first in human" Machbarkeitsstudie (einer Phase-I-Studie entsprechend) bei 45 Patienten mit therapieresistenter Hypertonie publiziert. Auch mit diesem System konnten systolischer und diastolischer Blutdruck im Mittel nach drei Monaten um 21/12 mmHg gesenkt werden. Die Implantation dieses Systems ist allerdings werder trivial noch frei von Komplikationen.

Patienten mit möglicher Indikation oder Behandlungswunsch sollten spezialisierten Zentren zugewiesen und vorzugsweise nur innerhalb von Studien behandelt werden. Zuvor ist es nach meiner Meinung wichtig enten eine sekundäre Hypertonie sowie häufige Ursachen der Therapieresistenz wie NonCompliance oder ein stark erhöhter Kochsalzkonsum nicht vorliegen.

Das Gespräch führte Jochen Aumiller II nachzuweisen, dass bei entsprechenden Pati-

Zielwerte bei der Hochdrucktherapie

Eigentlich gar nicht so kompliziert

„Viel wird diskutiert, welche Zielwerte bei der Drucksenkungen erreicht werden sollten. Aber eigentlich ist die aktuelle Lage gar nicht so kompliziert. Entscheidend ist, dass bei den meisten Hypertonikern der Zielblutdruck $<140 / 90 \mathrm{mmHg}$ eingestellt wird und die Diabetiker besonders gut im Zielkorridor $130-139 / 80-85 \mathrm{mmHg}$ kontrolliert werden. Eine Übertherapie mit Absenkung $<120 / 70 \mathrm{mmHg}$ soll bei Patienten mit nachgewiesener KHK vermieden werden. Die Diskussion um zu niedrige Blutdruckeinstellungen bei Hypertonikern soll aber nicht von unserer eigentlichen Herausforderung ablenken, dass immer noch ein Großteil der Hypertoniker einen nicht kontrollierten Blutdruck von systolisch über $\geq 140 \mathrm{mmHg}$ aufweist."

R. Kreutz II 